University of Montana

ScholarWorks at University of Montana

6-1-2003

\title{
Thickness Dependence of Magnetic Blocking in Granular Thin Films with Interacting Magnetic Particles
}

Jian Qing Wang

University of New Orleans

Zhi Dong Zhao

University of New Orleans

Scott L. Whittenburg

University of Montana - Missoula, scott.whittenburg@umontana.edu

Follow this and additional works at: https://scholarworks.umt.edu/chem_pubs

Part of the Biochemistry Commons, Chemistry Commons, and the Physics Commons Let us know how access to this document benefits you.

\section{Recommended Citation}

Wang, Jian Qing; Zhao, Zhi Dong; and Whittenburg, Scott L., "Thickness Dependence of Magnetic Blocking in Granular Thin Films with Interacting Magnetic Particles" (2003). Chemistry and Biochemistry Faculty Publications. 65.

https://scholarworks.umt.edu/chem_pubs/65

This Article is brought to you for free and open access by the Chemistry and Biochemistry at ScholarWorks at University of Montana. It has been accepted for inclusion in Chemistry and Biochemistry Faculty Publications by an authorized administrator of ScholarWorks at University of Montana. For more information, please contact scholarworks@mso.umt.edu. 


\title{
Thickness dependence of magnetic blocking in granular thin films with interacting magnetic particles
}

\author{
Jian-Qing Wang ${ }^{\text {a) }}$ \\ Department of Physics, State University of New York, Binghamton, New York 13902 \\ Zhi-Dong Zhao and Scott. L. Whittenburg \\ Advanced Materials Research Institute, University of New Orleans, New Orleans, Louisiana 70148
}

(Received 3 December 2002; accepted 10 March 2003)

\begin{abstract}
Interparticle interaction among single domain nanosize magnetic particles embedded in nonmagnetic matrix was studied. Attention was paid to concentrated $\mathrm{Cu}-\mathrm{Co}$ granular thin films with a fixed magnetic volume fraction (20\%). By analyzing theoretical models and comparing with experimental results, a dimensional constraint on the magnetic properties was found. As the film thickness reduces toward the thin limit the interparticle interaction plays important roles in modifying the magnetic behavior. The dipolar interaction energy was calculated among magnetic particles including far-neighbor interaction for films with different thickness values. When magnetization variation is included in the calculation, the resulting calculated interaction energy versus film thickness shows remarkable agreement with the variation of experimental observed peak temperature derived from magnetic blocking curves. (c) 2003 American Institute of Physics.
\end{abstract}

[DOI: $10.1063 / 1.1569993]$

\section{INTRODUCTION}

One of the main challenges in the era of information technology is the miniaturization of magnetic data bits into the nanoregime without losing stability of the stored information. ${ }^{1,2}$ This requires understanding interaction mechanisms between magnetic particles and developing materials ${ }^{3}$ with more stable magnetic states at reduced dimensions. Thus, there has been extensive recent interest in better understanding magnetic properties of heterogeneous materials with high concentrations of magnetic nanoparticles.

The main source of magnetic instability is related to the superparamagnetism at the ambient temperature. The superparamagnetic phenomenon is associated with the wellknown features in the dc susceptibility of systems with single domain magnetic particles, exhibiting a broad peak versus temperature. This phenomenon is a result of freezing magnetic moments below a characteristic temperature, $T_{B}$, called the blocking temperature. ${ }^{4,5}$ Above the blocking temperature thermal agitation overcomes the energy barriers pinning the magnetic moments, resulting a loss of magnetic memory. Below $T_{B}$, the energy of thermal excitations is too low to overcome energy barriers to rotate the magnetic moments of ultrafine particles within the characteristic time of the measurement.

The mechanisms for magnetic blocking are manifolds in a heterogeneous system, because there are different magnetic interactions that make blocking barriers for the magnetic moments of ultrafine particles. There are magnetic anisotropies that are always present in magnetic materials, including size, shape, stress, and crystalline anisotropy. In heterogeneous

a) Author to whom correspondence should be addressed; electronic mail: jqwang@binghamton.edu materials with concentrated magnetic particles the magnetostatic interaction is not negligible and, thus, could contribute to the interaction energy.

It is in this context we focus attention on the effects of magnetostatic interactions on magnetic blocking in granular magnetic thin films. The model system is a series of $\mathrm{Cu}_{80} \mathrm{Co}_{20}$ granular thin films with a fixed volume fraction of Co of $20 \%$. The system variation was realized by changing the film thickness over a wide range from 7 to $400 \mathrm{~nm}$. We studied magnetic dipole interactions among Co magnetic particles as the film thickness was varied. In this work, we report agreement obtained between the calculated dipoledipole interaction energy and the experimentally observed variation of magnetic blocking temperature versus the film thickness over the range of thickness studied.

\section{THICKNESS DEPENDENCE OF MAGNETIC PROPERTIES: EXPERIMENT}

First, we briefly summarize the main experimental results. Certain details were presented in a previous letter. ${ }^{6}$ Granular thin films of $\mathrm{Cu}_{80} \mathrm{Co}_{20}$ with nominal $20 \%$ Co were consistently deposited on $\mathrm{Si}$ substrates by magnetron sputtering in a high vacuum system. Constant magnetic composition and deposition conditions were maintained to achieve similar microstructure. A series of such films was deposited with different film thickness values and they were measured for their magnetic characteristics to study the thickness dependence of magnetic properties.

Microstructure characterizations were done on these granular thin films using transmission electron microscopy (TEM) and x-ray diffraction. It was found from TEM crosssection imaginary analysis that the granular films are continuous with smooth surfaces even down to the thinnest films studied $(7 \mathrm{~nm})$, as shown in Fig. 1 by TEM cross-section 


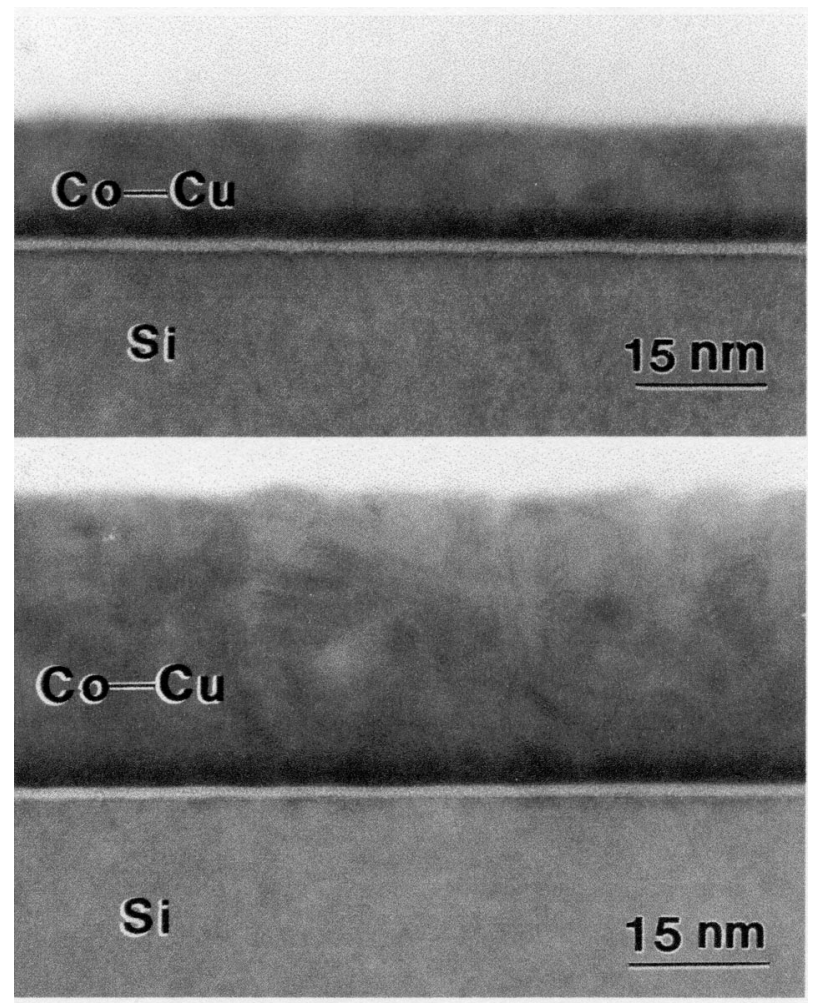

FIG. 1. Cross-sectional images of two granular films, with two different thickness values in very thin limit, show that the films are smooth and continuous.

images for two thinner $\mathrm{Cu}_{80} \mathrm{Co}_{20}$ films. Thus, it is unlikely that any observed magnetic effects and the related thickness dependence are due to surface roughness. Such roughened surfaces may induce Néel coupling which may create opposite magnetic poles adjacent to each other that affects the magnetic properties.

The magnetic microstructure was determined by measuring the magnetic properties (superparamagnetism or CurieWise behavior) to derive the size of magnetic clusters (or particles). It was found that the average particle size of Co particles was approximately $3 \mathrm{~nm}$ and was nearly constant with film thickness. ${ }^{6}$ The magnetic measurements were done in a superconducting quantum interference device magnetometer (Quantum Design) in a wide temperature range (from 4.2 to $400 \mathrm{~K}$ ) and field range (from -50 to $+50 \mathrm{Oe}$ ). Magnetization hysterisis loops were measured at various temperatures in a sweeping field between -50 to +50 Oe. Zerofield cooled (ZFC) and field-cooled (FC) magnetic susceptibilities were measured versus temperature. The susceptibility measurements were done in a small magnetic field (typically $50 \mathrm{Oe}$ ) to obtain strong enough magnetic signal and yet small enough that the measured susceptibility is within the linear region versus the magnetic field.

Representative results of measured dc ZFC and FC susceptibility curves are presented in Fig. 2 (also shown in Ref. 6), for films with two different thickness values, demonstrating typical behavior of superparamagnetism and magnetic blocking with a broad peak at some characteristic temperature $T_{M}$, very close to the blocking temperature. In fact, for the thicker films, $T_{M}$ coincides with $T_{B}$. By definition, the

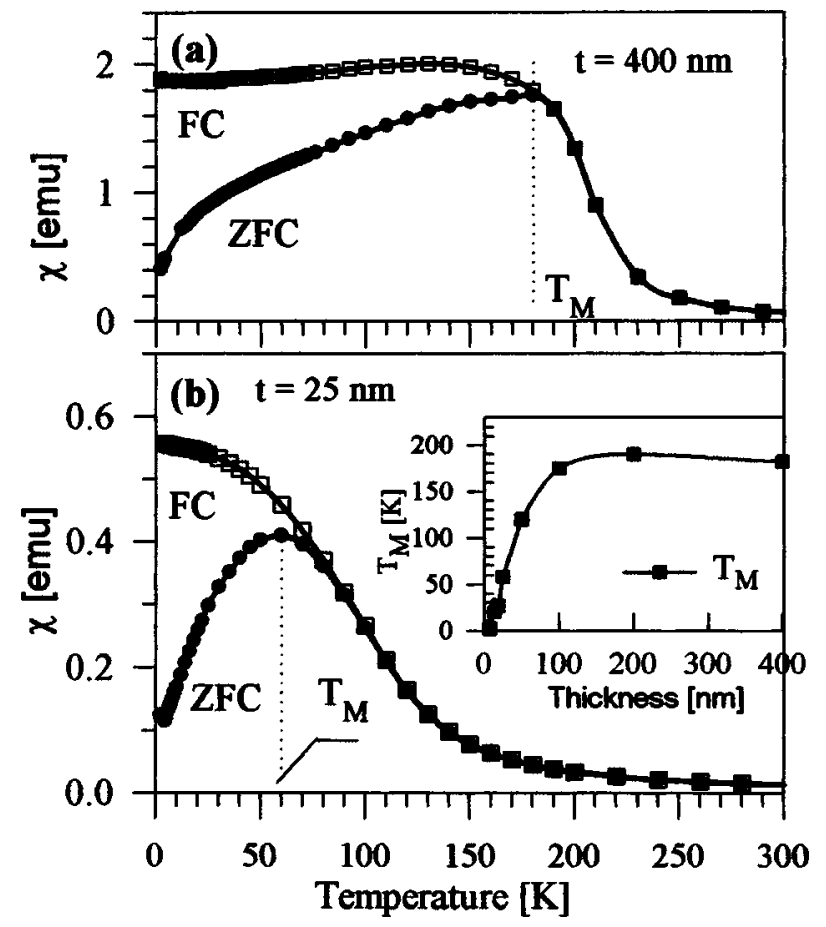

FIG. 2. The dependencies of ZFC and FC susceptibilities on the temperature for $\mathrm{Co}_{20} \mathrm{Cu}_{80}$ films with different thickness values: (a) $t=400 \mathrm{~nm}$ and (b) $t$ $=25 \mathrm{~nm}$. Inset: The thickness dependence of the peak temperature of the blocking curves vs film thickness for a series of $\mathrm{Co}_{20} \mathrm{Cu}_{80}$ granular films.

blocking temperature, $T_{B}$, is the minimum temperature above that the single domain particles become superparamagnetic, and the ZFC and FC susceptibility coincide with each other above $T_{B}$.

The first important fact to notice is that the blocking temperature for the thicker film is relatively high at $190 \mathrm{~K}$. This is substantially higher than in most of the granular films studied so far. The relatively high $T_{M}$ is unusual for as deposited films with ultrafine particles. Blocking temperature is directly proportional to the energy barrier for the magnetic moment of the particles to overcome by thermal agitation leading to superparamagnetism. Conventional analysis of $T_{M}$ generally considers contributions only from anisotropy energy. ${ }^{5,7}$ It is apparent that such high values of $T_{M}$ cannot be accounted for using only anisotropy consideration. According to conventional theory, $T_{B}$ is proportional to the product of anisotropy constant, $K$, and the particle volume, $V$, as $T_{B}=K V / 30 k_{B}$, where $k_{B}$ is the Boltzmann constant. From the known value ${ }^{8}$ of $K$ for the bulk Co, 2.7 $\times 10^{6} \mathrm{erg} / \mathrm{cm}^{3}$, and the measured Co particle size, $2.8 \mathrm{~nm}$, the estimated $T_{B}$ is only about $7 \mathrm{~K}$, in rough agreement with the measured value ${ }^{9} \sim 20 \mathrm{~K}$ for a powder sample composed of loosely bound Co nanoparticles. Thus, another interaction has to be considered to account for the high $T_{M}$ in these samples. Interparticle interaction plays a crucial role in enhancing the magnetic firmness and, thus, is an obvious candidate.

A second interesting feature in these samples is that $T_{M}$ is very different for the thicker and thinner films. In fact, below $100 \mathrm{~nm}$ the observed $T_{M}$ monotonically decreases with decreasing temperature, as shown in the inset of Fig. 
2(b) where we plot the dependence of $T_{M}$ on the film thickness. We observe a dramatic reduction of $T_{M}$ with decreasing thickness, $t$, of the granular films. The peak temperature changes by almost two orders of magnitude in a wide thickness range between 7 and $100 \mathrm{~nm}$, which shows that the interaction causing the finite size effect is long range in nature. It is plausible that interparticle interactions are responsible for and manifested in the thickness dependence of $T_{M}$ as well. The influence of the thickness on the interaction energy in a granular film with interacting particles can be qualitatively understood, because the number of neighboring interacting particles for particles in specific locations in the films decreases with decreasing thickness.

Among long-range effects dipole-dipole interaction is the dominant contribution, although other interactions may contribute. For example, the Ruderman-Kittel-(Kasuya)Yosida interaction responsible for the exchange coupling in $\mathrm{Co} / \mathrm{Cu}$ multilayers is likely to exist in $\mathrm{Cu}-\mathrm{Co}$ granular system. However, due to arbitrary shape and three-dimensional randomness of the particles, the strength of the interaction should be drastically reduced compared to multilayer films. This indicates that, to a large extend, the observed thickness dependence of magnetic blocking is due to dimensional constraint on the interparticle interactions. In fact, further detailed model analysis, as shown later, demonstrates that the magnetic dipole-dipole interaction is responsible for the high $T_{M}$ values and for the thickness dependence of the blocking temperature.

\section{MODEL ANALYSIS}

Quantitative evaluation of the energy barrier, $E_{\text {int }}$, on a magnetic particle by dipolar interactions of the neighboring particles can be calculated using the following relation as previously proposed: ${ }^{10}$

$$
E_{\mathrm{int}} \cong M^{2} V \sum_{i} n_{i} b_{i} L\left(M^{2} \frac{V a_{i}}{k_{B} T}\right), \quad i=1,2,3, \ldots
$$

where $n_{i}$ 's are the numbers of the $i$ th nearest neighbors of the interacting particles with magnetization $M$. The coefficients, $a_{i}$ 's and $b_{i}$ 's, are comparable to each other, and are given by the formula $a_{i}=V\left(3 \cos ^{2} \alpha_{i}-1\right) / d_{i}^{3}$; they are dependent on the distances $d_{i}$ 's and the position angles $\alpha_{i}$ 's between particles. The Langevin function, $L(x)$, demonstrates that the magnetic particles are in superparamagnetic state and contributions from other interacting sources are negligible.

To facilitate calculation, the earlier summations over discrete neighboring particles with different distances can be converted into integrations with continuous space variables. For example, at the center of a film with a thickness value, $t$, interaction energy can be expressed in the following form:

$$
\begin{aligned}
E_{\mathrm{int}} \cong & M^{2} V^{2}\langle\rho\rangle 2 \pi\left[\int_{-t / 2}^{t / 2} \int_{\delta}^{r_{\max }}+\left(\int_{-t / 2}^{\delta^{\prime}}+\int_{\delta^{\prime}}^{t / 2}\right) \cdot \int_{0}^{\delta}\right] \\
& \times \frac{r\left(3 \cos ^{2} \alpha-1\right)}{\left(r^{2}+\zeta^{2}\right)^{3 / 2}} L\left[\frac{M^{2} V^{2}\left(3 \cos ^{2} \alpha-1\right)}{k_{B} T\left(r^{2}+\zeta^{2}\right)^{3 / 2}}\right] d r d \zeta,
\end{aligned}
$$

where $\langle\rho\rangle$ represents the mean density of magnetic particles and the integration excludes a small sphere with characteris-

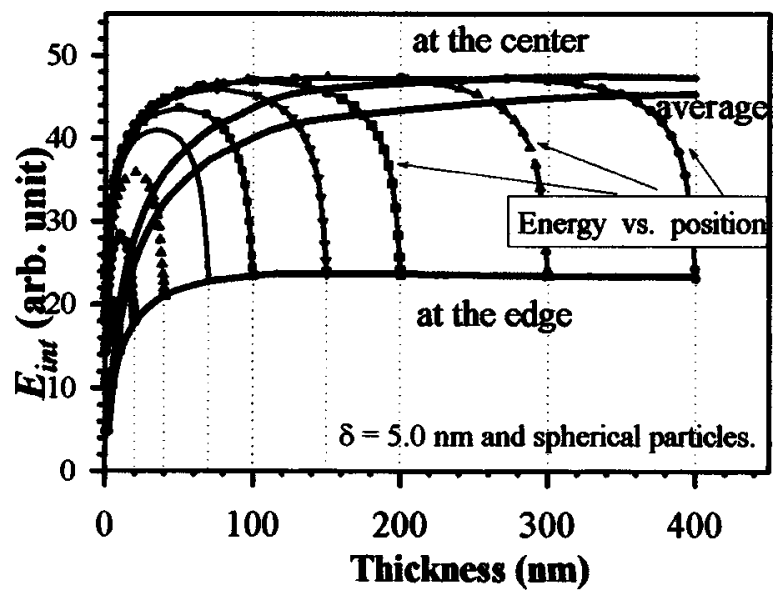

FIG. 3. Calculated dipolar interaction energy, $E_{\text {int }}$, at various locations for films of different thickness values. Each line with a particular symbol represents an energy curve across a particular film with a thickness value at the end of that curve. Also shown are the interaction energies (thicker curves) at the edge and center, and the average energy as a function of film thickness, respectively.

tic radius $\delta$, corresponding to the distance between nearest neighbors; $\delta^{\prime}=\sqrt{\left(\delta^{2}-r^{2}\right)}$ for $r \leqslant \delta$. In fact, the discrete nature of the magnetic particles is reflected by such a void in the integration, beyond which the magnetic particle distribution is treated as continuous. In the cylindrical coordinate $\zeta$ is related to the thickness, $t$, and $\cos \alpha$ is a function of $r$ and $\zeta$ : $\cos \alpha=r /\left(r^{2}+\zeta^{2}\right)^{1 / 2}$.

The upper limit, $r_{\max }$, in the integration in the film plan, can be understood as some realistic physical constraints or typical domain size formed by magnetic particles. The existence of magnetic domains in granular films was demonstrated by recent studies. ${ }^{11,12}$ Even though single domain and nanosized ferromagnetic particles are physically separated from each other, through certain interactions, magnetic particles form ferromagnetic domains with typical domain sizes of about 100-140 nm. Thus, one expects that beyond such length scale dipolar interactions diminish with distance and can be neglected. The estimated dipolar interaction strength within such a domain provides sufficient energy corresponding to a $T_{M}$ value of about $100 \mathrm{~K}$.

Results of integration using the above simple model are presented in Fig. 3 for energy variation distributed across the granular films with different thickness values. The calculated average energy (thick solid line, with $\delta=5 \mathrm{~nm}$ ) indicates that around $100 \mathrm{~nm}$ the energy barrier related to dipolar interaction begins to decrease, first gradually and then more dramatically, which could account for the experimental trends. However, the detailed shape of the energy curves does not exactly match the experimentally observed variation of blocking temperature. The decrease in the experimentally observed $T_{M}$ is more abrupt below $100 \mathrm{~nm}$, following almost linear decreasing behavior, while the calculated energy curve is more gradual at the beginning. Nonetheless, the calculated energy curves all approach zero as $t$ approaches zero, correctly predicting the experimental observation.

Upon further examination of the experimental results, we found that the spontaneous magnetization, $M_{S}$ of these 


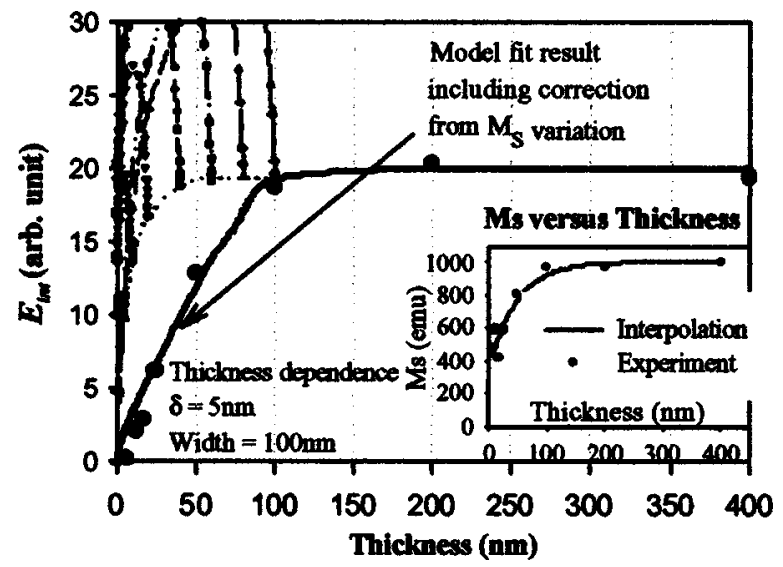

FIG. 4. Comparison of the measured peak temperature, $T_{M}$, vs film thickness with the calculated dipolar interaction, $E_{\text {int }}$, corrected for the variation of spontaneous magnetization, $M_{S}$. The model calculated energy uses the edge energy curve with the assumption that magnetic blocking reflects the lowest energy barrier. Inset: measured $M_{S}$ vs film thickness.

granular films does not maintain a constant value as a function of film thickness. The variation of $M_{S}$ is shown in the inset of Fig. 4, showing that the value of $M_{S}$ starts to decrease below $100 \mathrm{~nm}$ and it reaches about half the value for the thicker films in the thin limit. This indicates that for thinner films there was incomplete phase separation between $\mathrm{Co}$ and $\mathrm{Cu}$ atoms in the Co-rich clusters, since there is a small miscibility (a few percent) between the two elements. In the nanophase alloys, atomically mixing of $\mathrm{Co}$ and $\mathrm{Cu}$ causes the Co clusters to lose magnetic moment and become nonmagnetic. Also, the $\mathrm{Co}-\mathrm{Cu}$ interface reduces the magnetization due to magnetic dead layer. This is evident in the thicker films with lower value (1000 emu) of $M_{S}$ compared to bulk Co $(1400 \mathrm{emu})$.

It is likely that there is an accumulative heat dissipation effect due to kinetic bombardment of sputtering during the deposition of the films, causing a slightly higher temperature during the deposition in the thicker films resulting in better phase separation. A lower magnetic moment per particle means a lower interaction energy. In fact, the interaction energy, $E_{\text {int }}$, which is a two-particle interaction type, should scale as $M_{S}^{2}$ in addition to the finite-size dependence demonstrated earlier. When this correction is taken into account there is a quantitative agreement between the calculated in- teraction energy curve and the observed $T_{M}$ as a function of thickness, as shown in Fig. 4. In Fig. 4, the $E_{\text {int }}$ curve on the edge of the granular films is compared with the measured $T_{M}$, showing an excellent agreement over the whole thickness range studied. The reason for using the edge $E_{\text {int }}$ for comparison is due to the fact that $T_{M}$ measures the minimal energy barrier for blocking of the magnetic moments of the particles, and at the edges of the film the interaction energy is the lowest.

\section{CONCLUSIONS}

Interparticle interaction among single domain nanosize magnetic particles embedded in nonmagnetic matrix was studied for $\mathrm{Cu}_{80} \mathrm{Co}_{20}$ granular thin films. By analyzing theoretical models and comparing with experimental results, we found that the interparticle dipolar interaction plays important roles in modifying magnetic behavior and magnetic blocking. The calculation revealed that the interaction energy varies across the film from edge to edge and it is strongly dependent on film thickness. Good quantitative agreement of the calculated energy curve with the experimental blocking energy derived from magnetic blocking curve was achieved after taking the magnetization variation into account.

\section{ACKNOWLEDGMENTS}

The authors would like to thank Dr. Serif Uran and Dr. Weilie Zhou for technical assistance. This work was supported by DoD/DARPA MDA972-97-1-0003.

${ }^{1}$ M. H. Kryder, MRS Bull. 21, 17 (1996).

${ }^{2}$ E. D. Dahlberg and J.-G. Zhu, Phys. Today 48, 34 (1995).

${ }^{3}$ S. Sun, C. B. Murray, D. Weller, L. Folks, and A. Moser, Science 287, 1989 (2000).

${ }^{4}$ L. Néel, Acad. Sci. 228, 664 (1949).

${ }^{5}$ W. F. Brown, Phys. Rev. 130, 1677 (1963).

${ }^{6}$ L. M. Malkinski, J.-Q. Wang, J. Dai, J. Tang, and C. J. O'Connor, Appl. Phys. Lett. 75, 844 (1999).

${ }^{7}$ J. I. Gittleman, B. Abeles, and S. Bozowski, Phys. Rev. B 9, 3891 (1974).

${ }^{8}$ W. D. Doyle and P. J. Flanders, International Conference on Magnetism (Nottingham, 1964, IOP, Bristol, 1965), p. 751.

${ }^{9}$ J. P. Chen, C. M. Sorensen, K. J. Klabunde, and G. C. Hadjipanayis, J. Appl. Phys. 76, 6316 (1994).

${ }^{10}$ J. L. Dormann, L. Bessais, and D. Fiorani, J. Phys. C 21, 2015 (1988).

${ }^{11}$ A. Gavrin, M. H. Kelley, J. Q. Xiao, and C. L. Chien, Appl. Phys. Lett. 66, 1683 (1995)

${ }^{12}$ Y. J. Chen, W. Y. Cheung, I. H. Wilson, N. Ke, S. P. Wong, and J. B. Xu, Appl. Phys. Lett. 72, 2472 (1998). 